\title{
Animal models of sepsis and sepsis-induced kidney injury
}

\author{
Kent Doi, ${ }^{1}$ Asada Leelahavanichkul, ${ }^{2,3}$ Peter S.T. Yuen, ${ }^{2}$ and Robert A. Star ${ }^{2}$
}

${ }^{1}$ Department of Nephrology and Endocrinology, University of Tokyo, Tokyo, Japan. ${ }^{2} \mathrm{NIDDK}$, NIH, Bethesda, Maryland, USA. ${ }^{3}$ Division of Nephrology, Department of Medicine, Chulalongkorn University, Bangkok, Thailand.

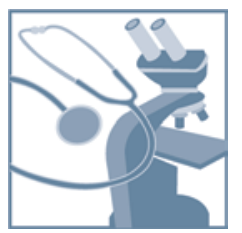

Sepsis is characterized by a severe inflammatory response to infection, and its complications, including acute kidney injury, can be fatal. Animal models that correctly mimic human disease are extremely valuable because they hasten the development of clinically useful therapeutics. Too often, however, animal models do not properly mimic human disease. In this Review, we outline a bedside-to-bench-to-bedside approach that has resulted in improved animal models for the study of sepsis - a complex disease for which preventive and therapeutic strategies are unfortunately lacking. We also highlight a few of the promising avenues for therapeutic advances and biomarkers for sepsis and sepsis-induced acute kidney injury. Finally, we review how the study of drug targets and biomarkers are affected by and in turn have influenced these evolving animal models.

\section{Sepsis and sepsis-induced acute kidney injury: a life-threatening condition}

Sepsis is a characteristic set of systemic reactions to overwhelming infection. Sepsis, severe sepsis, and septic shock are defined according to established criteria (Table 1) (1). Discovery of antibiotics has dramatically improved the morbidity and mortality of the infectious diseases for the last decades; indeed, antibiotics and volume resuscitation are the first line of sepsis treatment strategy (2). However, overwhelming inflammatory response accompanied by depression in immunological function causes multiple organ injury and determines clinical outcomes. In addition to inflammation and immunological dysregulation, a number of different mechanisms contribute to sepsis at different phases (Figure 1). For instance, systemic hemodynamics evolves from an early hyperdynamic ("warm shock") state to a late hypodynamic ("cold shock") state.

Sepsis is the leading cause of death in critically ill patients, and the incidence of sepsis is increasing $(3,4)$. The mortality rate of severe sepsis is very high (up to $70 \%$ ), and the calculated costs exceed $\$ 15$ billion per year in the United States (3). The rate of severe sepsis during hospitalization almost doubled during the last decade and is considerably greater than previously predicted (5). Sepsis causes multiorgan failure, including acute kidney injury (AKI) (6), and patients with both sepsis and AKI have an especially high mortality rate (7). AKI is diagnosed by a sudden decrease in glomerular filtration rate (GFR), the primary measure of kidney function, which is currently detected clinically as a rise in serum creatinine. A multinational prospective observational study including 29,269 critically ill patients revealed that the occurrence of AKI in the intensive care unit (ICU) was approximately $6 \%$, the most frequent contributing factor to AKI being sepsis (50\%) (8). Other reports showed that between $45 \%$ and $70 \%$ of all AKI is associated with sepsis (9-11). Several different pathophysiological mechanisms have been proposed for sepsis-induced AKI: vasodilation-induced glomerular hypoperfusion, dysregulated circulation within the peritubular capillary network, inflammatory reactions by systemic cytokine storm or local cytokine production (12), and tubular dysfunction induced by oxidative stress (13).

Conflict of interest: The authors have declared that no conflict of interest exists. Citation for this article: J. Clin. Invest. 119:2868-2878 (2009). doi:10.1172/JCI39421.
Continuing concern over the efficacy and safety of the only FDAapproved therapy for severe sepsis (activated protein C) highlights the critical need to improve our understanding of the pathophysiology of sepsis and sepsis-induced AKI and to develop novel treatment strategies for critically ill patients (14). A multitude of potential drug targets have been identified in animal models of sepsis; however, translation from animals to humans has been exceedingly difficult. Several reviews have pointed out that the failure to translate results from animals to humans has been attributed to disease characteristics of sepsis (complexity and heterogeneity), inappropriate clinical trials (study of ineffective drugs, inadequate clinical trial designs), and animal models that do not fully mimic human sepsis (14-17).

\section{Requirements for animal models of sepsis}

Human sepsis is currently hypothesized to involve at least two stages: an initial proinflammatory burst responsible for hypotension and organ dysfunction, followed by a compensatory antiinflammatory immune response that leads to an immunosuppressed state often called immune depression or immune dysfunction; however, these stages can overlap temporally (Figure 1). The latter consists of altered monocyte antigen presentation, decreased lymphocyte proliferation and responsiveness, and lymphocyte apoptosis and anergy $(18,19)$, which accounts for nosocomial infections and late deaths in sepsis. Animal models of sepsis need to reproduce the complexity of human sepsis and its treatment in the ICU. Ideally, animal models should mimic the pace and severity of human sepsis; reproduce key hemodynamic (warm shock followed by cold shock) and immunologic (proinflammatory stimulation, antiinflammatory counterregulation, i.e., immune depression) stages; mimic histology findings in key organs (lung, liver, spleen, kidney, etc.) that are frequently modest; and - perhaps counterintuitively for animal modelers - exhibit variability among animals.

\section{Standard animal models of sepsis}

Sepsis animal models can be divided into three categories: (a) injection of an exogenous toxin (e.g., LPS); (b) alteration of the animal's endogenous protective barrier, such as intestinal leakage (e.g., cecal ligation and puncture [CLP] or colon ascendens 


\section{Table 1}

Sepsis definitions

Condition
SIRS

Sepsis

Severe sepsis

Septic shock

Refractory septic shock

\section{Description}

Two or more of the following conditions: temperature $>38.5^{\circ} \mathrm{C}$ or $<35.0^{\circ} \mathrm{C}$; heart rate of $>90$ beats $/ \mathrm{min}$; respiratory rate of $>20$ breaths $/ \mathrm{min}$ or $\mathrm{PaCO}_{2}$ of $<32 \mathrm{mmHg}$; and wbc count of $>12,000$ cells $/ \mathrm{ml},<4,000$ cells $/ \mathrm{ml}$, or $>10 \%$ immature (band) forms

SIRS in response to documented infection (culture or Gram stain of blood, sputum, urine, or normally sterile body fluid positive for pathogenic microorganism; or focus of infection identified by visual inspection)

Sepsis and at least one of the following signs of organ hypoperfusion or organ dysfunction: areas of mottled skin; capillary refilling of $>3 \mathrm{~s}$; urinary output of $<0.5 \mathrm{ml} / \mathrm{kg}$ for at least $1 \mathrm{~h}$ or renal replacement therapy; lactate of $>2 \mathrm{mmol} / \mathrm{l}$; abrupt change in mental status or abnormal EEG; platelet count of $<100,000$ cells/ml or disseminated intravascular coagulation; acute lung injury/ARDS; and cardiac dysfunction (echocardiography)

Severe sepsis and one of the following conditions: systemic mean BP of $<60 \mathrm{mmHg}(<80 \mathrm{mmHg}$ if previous hypertension) after 20-30 ml/kg starch or 40-60 ml/kg serum saline solution or PCWP between 12 and $20 \mathrm{mmHg}$; and need for dopamine of $>5 \mu \mathrm{g} / \mathrm{kg} / \mathrm{min}$, or norepinephrine or epinephrine of $<0.25 \mu \mathrm{g} / \mathrm{kg} / \mathrm{min}$ to maintain mean BP at $>60 \mathrm{mmHg}$ ( $80 \mathrm{mmHg}$ if previous hypertension)

Need for dopamine at $>15 \mu \mathrm{g} / \mathrm{kg} / \mathrm{min}$ or for norepinephrine or epinephrine at $>0.25 \mu \mathrm{g} / \mathrm{kg} / \mathrm{min}$ to maintain mean BP of $>60 \mathrm{mmHg}$ (80 $\mathrm{mmHg}$ if previous hypertension)

SIRS, systemic inflammatory response syndrome; ARDS, acute respiratory distress syndrome; PCWP, pulmonary capillary wedge pressure.

stent peritonitis [CASP]); and (c) infusion or instillation of exogenous bacteria (Table 2).

LPS-induced inflammation models. Endotoxin, a component of the outer membrane of Gram-negative bacteria, is involved in the pathogenesis of sepsis, and an LPS infusion/injection model has been widely used for sepsis research. LPS administration induces systemic inflammation that mimics many of the initial clinical features of sepsis, including increases in proinflammatory cytokines such as TNF- $\alpha$ and IL-1, but without bacteremia (20-22). Treatment of LPS-injected animals with neutralizing antibody against TNF- $\alpha$ or IL- 1 resulted in improved outcomes for this model (23, 24). A case report describes a patient who self-administered a large dose of LPS, and the full clinical manifestations of septic shock developed (25). LPS infusion also causes renal injury, including decreased GFR, increased blood urea nitrogen (BUN), and increased renal neutrophil infiltration (26-28).

Several clinical trials of anti-TNF- $\alpha$ and anti-IL-1 therapy were performed based on the promising results in LPS animal studies; however, these trials failed to improve survival of septic patients $(29,30)$. LPS causes much earlier and higher peak levels of cytokine expression compared with levels observed in human sepsis, with the notable exception of meningococcal sepsis, a rare, pathogen- and site-specific form of sepsis wherein cytokine levels are comparable to those observed in LPS animal models $(21,31,32)$. Also, some features of LPS infusion such as renal hypoperfusion and increased BUN are alleviated by volume replacement, which is routinely performed in clinical management of sepsis $(33,34)$. Nevertheless, LPS infusion remains a useful tool for interrogating a simpler subset of the complex trajectory of sepsis. The LPS dose can be titrated to mimic early sepsis without hemodynamic compromise, which has been useful for studying systemic and renal responses during the initial phases of sepsis; doses of LPS typically used induce systemic hypotension and decrease glomerular perfusion, whereas lower doses of LPS do not cause any systemic hypotension but still decrease glomerular perfusion $(35,36)$.

CLP of polymicrobial sepsis. CLP is currently the most widely used animal model of sepsis $(15,37,38)$. CLP surgery is straightforward: ligation distal to the ileocecal valve and needle puncture of ligated cecum cause leakage of fecal contents into the peritoneum, with subsequent polymicrobial bacteremia and sepsis (39). This surgical manipulation, while not well standardized, allows the severity to be adjusted by the length of ligated cecum and the size and/or number of the puncture. Supportive treatment with fluids and antibiotics is quite variable across laboratories and almost always inadequate, since typically only a single fluid and/or antibiotic dose is given (40). Multiple species of bacteria are found in the bloodstream (41, 42), and progressive systemic inflammatory response syndrome followed by septic shock and multiorgan injury ensues $(34,43,44)$. Mice subjected to CLP generally became severely hypotensive without an apparent hyperdynamic phase (45), although more vigorous fluid resuscitation can result in an early hyperdynamic phase detected by echocardiography (46). CLP-induced sepsis models show a cytokine profile similar to that in human sepsis $(21,31,34)$, and anti-TNF- $\alpha$ treatment fails to alleviate sepsis in CLP models as in human sepsis $(31,47,48)$. Notably, treatment with recombinant human TNF- $\alpha$ reduced mortality in CLP-induced sepsis (41). As described above, human sepsis is considered to have two immunologically different stages: a proinflammatory phase and a compensatory antiinflammatory phase. CLP-induced sepsis increased lymphocyte apoptosis, which mimics immunosuppression at the later phase of human sepsis $(18,49,50)$. In this respect, CLP-induced sepsis is completely different from LPS-induced sepsis and more closely mimics human sepsis.

However, the standard CLP model does not develop reproducible acute kidney or lung injury. AKI has been detected by changes in BUN or creatinine in some (51-55) but not other studies $(56,57)$. Thus, the standard CLP model encompasses more clinical features and drug responses of human sepsis than the LPS model but is still missing some key features, especially kidney and lung injury.

Bacterial infusion or instillation models. Whereas models such as CLP and CASP are helpful in understanding polymicrobial sepsis, human sepsis may also be caused by a single pathogen. Bacterial infusion models can approximate introduction of a single pathogen in a controlled manner, allowing reproducible infection. These 


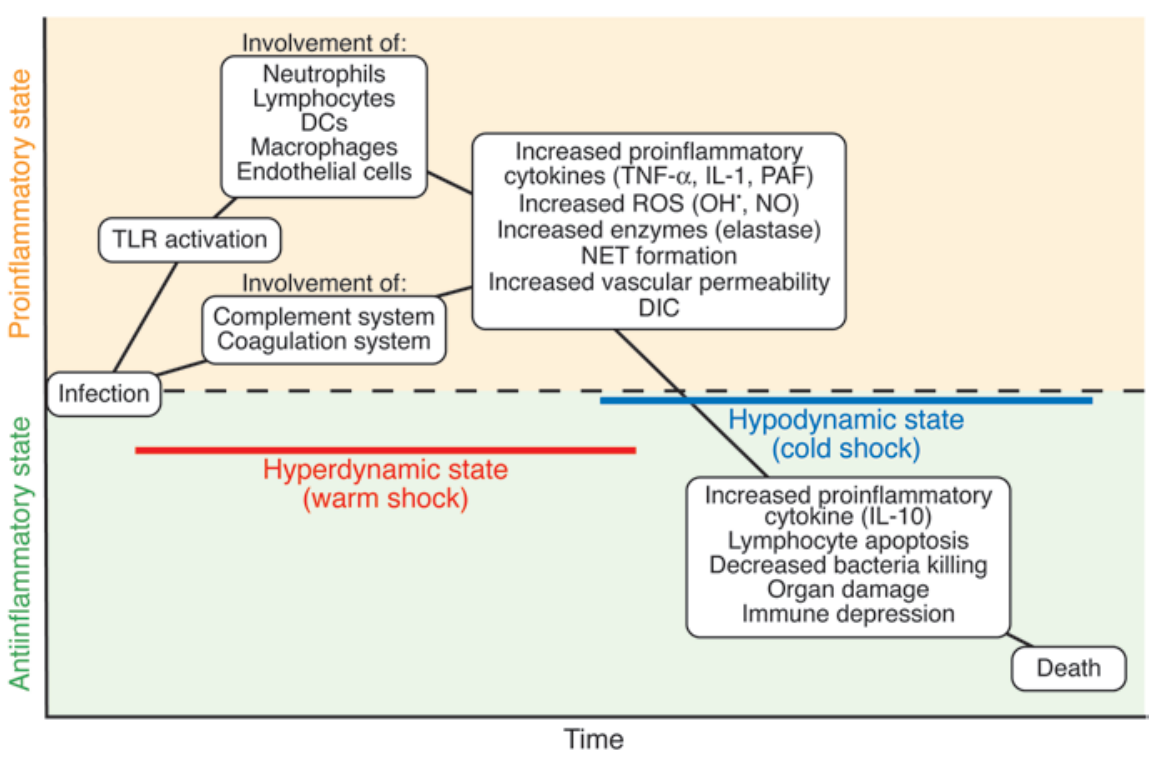

\section{Figure 1}

Simplified clinical course of sepsis. Progression of disease is complex, nonlinear, and varies from one patient to another. Shown is an outline of selected landmark events and processes that appear to be common among patients and some animal models. DIC, disseminated intravascular coagulation. models have been translated to larger animals for the study of systemic and organ-specific hemodynamics (see below). Similarly, instillation can be useful for simulating pneumonia, especially as a nosocomial infection (58). These models provide complementary information that is likely to be pathogen specific.

\section{Creating new clinically relevant sepsis models}

LPS and traditional CLP models mimic the clinical conditions of meningococcal sepsis or postsurgical peritonitis of young patients. However, animal models of sepsis differ from human sepsis because of age, comorbidity, and use of supportive therapy (16). Starting from a clinical perspective, we reasoned that within practical limitations, animals should receive treatment comparable to the supportive therapy that is standard for ICU patients. This is essential to enable us to test whether a therapy has additional benefit beyond that provided by conventional fluid and antibiotic therapy. We also hypothesized that the CLP model could be improved by simulation of one or more underlying baseline conditions typically present in septic patients, such as advanced age or chronic disease.

Age, resuscitation, and antibiotics. Since the incidence of sepsis dramatically increases with age, and elderly patients are especially prone to sepsis and sepsis-induced AKI (59), our first modification of standard CLP models was to employ older mice, i.e., retired breeders. We and others found that aged mice (16 to 50 weeks old) were more susceptible to CLP and LPS than young mice around 8 to 16 weeks old $(34,60,61)$. In standard mouse CLP models, animals are typically given a small amount of fluid resuscitation and perhaps a dose of antibiotics immediately after surgery, but fluid and antibiotic treatment are not continued. Because volume resuscitation can reverse LPS-induced renal injury in aged mice (34), we added volume resuscitation and antibiotic treatment to the standard CLP model. Animals became clinically ill at 5-6 hours after surgery, at which time they had evidence of liver damage; kidney damage was evident by MRI early (62), but serum creatinine and BUN levels did not significantly increase until 12 hours after surgery (63). With these additions, consistent histological renal damage with significant increase in serum creatinine were observed, not because fluids and antibiotics were harmful to the kidney, but because the animals could survive long enough to develop AKI (34, 64). Acute tubular necrosis is found in ischemic and toxic AKI but is not present in any of our mouse CLP models; in contrast, we observed areas of proximal tubular cells that contain prominent intracellular vacuoles of unknown composition (Figure 2). A recent systematic review of sepsis revealed that there were only mild histological changes in human and animal sepsis, and acute tubular necrosis was relatively uncommon (65). Among these studies, Sato et al. detected increased vacuolization and flattening of the brush border membrane in immediate autopsy of septic patients (66).

Table 2

Animal models of sepsis

$\begin{array}{lc}\text { Animal model } & \text { Advantage } \\ \text { LPS injection } & \begin{array}{c}\text { Simple, sterile; some similarities with } \\ \text { human sepsis pathophysiology }\end{array} \\ \text { CLP or CASP } & \begin{array}{c}\text { Early silent period; moderate and delayed peak } \\ \text { of mediators; multiple bacterial flora } \\ \text { Replication of clinical risk factors }\end{array} \\ \begin{array}{c}\text { Clinically relevant CLP } \\ \text { Infusion or instillation } \\ \text { of exogenous bacteria }\end{array} & \text { Early hyperdynamic state }\end{array}$

\section{Disadvantage}

Early and transient increases in inflammatory mediators more intense than in human sepsis

Age and strain variability; early hemodynamic period in some models

Difficulty in analyzing pathophysiological pathways

No change in intrarenal microcirculation; need large animals; labor-intensive 


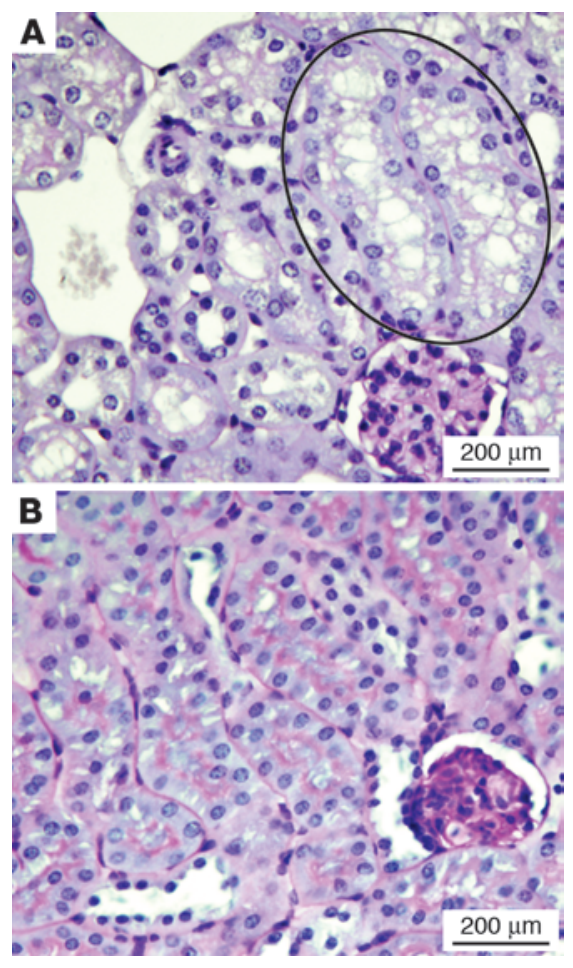

Although a similar tubular vacuolization is found in cyclosporine nephropathy (67), its significance and relevance in sepsis still need to be clarified (68).

Genetic heterogeneity. We next developed a CLP model in aged outbred rats (Sprague-Dawley strain). We originally switched to rats because serum and urine are difficult to collect from mice (44). In contrast to the mouse CLP model, which consistently develops AKI with multiorgan injury, the rat CLP model showed heterogeneous responses, with a wide spectrum of degrees of AKI, including clear histological evidence for AKI. This model demonstrated early but not late increases in IL-6 levels that corresponded to development of AKI, similar to those seen in septic patients (69).

Could the variability and sensitivity to AKI be attributed to the outbred Sprague-Dawley strain? We shifted our CLP mouse model to the outbred CD-1 strain and found that CD-1 mice developed sepsis AKI at a young age (64), whereas the inbred C57BL/6 strain developed AKI only at an advanced age (34). The reason for this difference in susceptibility is unknown, but the genetically heterogeneous human population should be more accurately represented by outbred mice, reducing the bias found in inbred strains that might contain or lack recessive disease susceptibility loci, depending on selective pressures $(70,71)$.

Predisposing comorbid conditions. Animal studies typically examine sepsis and related organ failure in otherwise healthy animals, despite numerous epidemiological studies of human sepsis that show the importance of preexisting comorbid conditions $(3,72)$. Severe sepsis occurs frequently in patients with underlying chronic diseases (comorbidities) including chronic kidney disease (CKD), liver disease, and diabetes and has an extremely high mortality rate $(3,72)$. CKD is found in approximately $30 \%$ of AKI patients in the ICU $(8,73)$. Patients with CKD also have an increased risk of morbidity and mortality from sepsis (74-77). These findings suggest that clinical sepsis and sepsis-induced AKI are dramati-

\section{Figure 2}

Histology of AKI in a clinically relevant model of CLP-induced sepsis. Periodic acid-Schiff (PAS) staining of mouse kidney cortex 24 hours after CLP surgery (A) or sham surgery (B). Pink staining of brush border is visible in sham cortical tubules, and loss of brush border is evident, as is mild dilation, after CLP. Vacuolization is seen after CLP in almost all tubules, most prominently in two tubules in the upper-right corner (circled). Original magnification, $\times 400$. Scale bar: $200 \mu \mathrm{m}$. Reproduced from the American Journal of Physiology: Renal Physiology (63).

cally influenced by underlying diseases, which may help explain why simple animal models of sepsis do not mimic human sepsis and do not predict human response to therapeutics. We recently established a two-stage mouse model of preexisting renal disease with subsequent sepsis (CKD-sepsis) to mimic the complexity of human sepsis; mice were given folic acid to induce renal fibrotic injury, then subjected two weeks later to CLP surgery (78). This CKD-sepsis model showed increased vascular permeability and decreased bacterial clearance compared with sepsis animals without the comorbidity of CKD. Combination therapy with soluble FMS-like tyrosine kinase 1 (FLT1; also known as VEGF receptor 1) and chloroquine, which block vascular and immunological dysfunction, respectively, resulted in the best survival rate. Both drugs were effective individually in simple CLP models, but neither drug alone was effective in the more complex CKD/CLP model, suggesting that multiple therapeutic interventions ("combination chemotherapy") may be required for the treatment of sepsis complicated with comorbidity. Complex animal models of human sepsis may be more pharmacologically relevant than simple animal models for the testing of therapeutics, as they may ultimately predict human drug responsiveness more accurately. The introduction in these models of other disease-modifying variables (e.g. advanced age, supportive treatment with fluids or antibiotics, or the presence of other chronic comorbidities) appears to alter and complicate the underlying pathophysiological mechanisms of disease. Therefore, it is important to compare the complex models with simpler models systematically to distinguish between core versus amplifying factors in sepsis, in hopes of discovering new methods to better classify septic patients into informative subgroups, each with a more uniform set of pathophysiological mechanisms.

Late-stage immunosuppression. Despite a focus on the proinflammatory aspects of sepsis, most deaths in sepsis occur from nosocomial infections during a late prolonged immunosuppressed state, even in the face of successful early, supportive therapies. Septic patients have defects in innate and adaptive immunity, including altered monocyte antigen presentation, decreased lymphocyte proliferation and responsiveness, and lymphocyte apoptosis and anergy $(18,19)$. Prevention and/or treatment of this immune deficiency should be a focal point for novel treatments; immunostimulatory therapies such as IFN- $\gamma$ and GM-CSF are being tested in early-phase clinical trials (79), However, this dimension of sepsis has been underrepresented in animal models; simple CLP models have splenic apoptosis but generally die too early before later immunosuppression fully develops. More complex models have been developed recently. A "two-hit" model of CLP followed by instillation of bacteria (Pseudomonas aeruginosa or Streptococcus pneumoniae) mimics nosocomial infections that result from immune depression $(58,80)$. In these animals, proinflammatory cytokines (e.g., IL-6) were decreased, antiinflammatory (e.g., IL-10) cytokines were increased, bacteria clearance was reduced, and profound 
Table 3

A partial list of emerging therapeutic targets tested in animal models

\begin{tabular}{|c|c|c|c|}
\hline Target & Primary mechanism & Agent & Ref. \\
\hline HMGB1 & $\begin{array}{l}\text { HMGB1 is released from damaged cells in sepsis } \\
\text { HMGB1 activates NF- } \mathrm{KB} \text { via RAGE, TLR2, TLR4 }\end{array}$ & Neutralizing Ab & S189 \\
\hline Lymphocyte apoptosis & $\begin{array}{l}\text { Apoptosis induces depletion of immune cells } \\
\text { Apoptosis impairs immunity by inducing anergy }\end{array}$ & $\begin{array}{l}\text { Caspase inhibitor } \\
\text { CD95 fusion protein/siRNA }\end{array}$ & S190 \\
\hline \multicolumn{4}{|l|}{ Pathogen recognition } \\
\hline TLR4 & E. coli sepsis induces cytokine production via TLR4 & Neutralizing Ab & 124 \\
\hline TLR9 & Bacterial DNA induces cytokine production via TLR9 & $\begin{array}{l}\text { ODN TLR9 inhibitor } \\
\text { Chloroquine }\end{array}$ & $\begin{array}{c}S 128 \\
42\end{array}$ \\
\hline \multicolumn{4}{|l|}{ End-organ damage } \\
\hline VEGF & $\begin{array}{l}\text { Systemic VEGF level increases in sepsis } \\
\text { VEGF-induced vascular leakage }\end{array}$ & Soluble Flt1 & $78, S 138, S 139$ \\
\hline C5a & $\begin{array}{l}\text { C5a induces lymphocyte apoptosis and coagulation system failure } \\
\text { C5a induces HMGB1 release via C5L2 }\end{array}$ & $\begin{array}{l}\text { Neutralizing } \mathrm{Ab} \text { to } \mathrm{C} 5 \mathrm{a} \\
\text { Neutralizing } \mathrm{Ab} \text { to } \mathrm{C} 5 \mathrm{aR}, \mathrm{C} 5 \mathrm{~L} 2\end{array}$ & $\begin{array}{l}\text { S142, S191 } \\
\quad \text { S141 }\end{array}$ \\
\hline \multicolumn{4}{|l|}{ Neuroimmune axis } \\
\hline $\begin{array}{l}\text { Parasympathetic } \\
\text { nerve system }\end{array}$ & $\begin{array}{l}\text { Vagus nerve stimulation attenuates an inflammatory response via } \alpha 7 n A C h R \\
\text { Acetylcholine inhibits HMGB1 release via } \alpha 7 n A c h R\end{array}$ & Nicotine & S147 \\
\hline $\begin{array}{l}\text { Pituitary hormones } \\
\alpha-\mathrm{MSH} \\
\text { Ghrelin }\end{array}$ & $\begin{array}{l}\alpha-M S H \text { decreases inflammatory cytokines and NO production } \\
\text { Ghrelin decreases HMGB1 release and has antibacterial activity }\end{array}$ & $\begin{array}{l}\alpha-M S H \text { analog } \\
\text { Ghrelin }\end{array}$ & $\begin{array}{c}64 \\
S 158\end{array}$ \\
\hline \multicolumn{4}{|l|}{ Cell-based therapy } \\
\hline NETs & $\begin{array}{l}\text { NETs trap and kill bacteria in blood and tissue } \\
\text { TLR4 activation induces NET formation }\end{array}$ & Eritoran (TLR4 antagonist) & S161 \\
\hline MSCs & MSC reprograms macrophage to produce IL-10 & MSC & S171 \\
\hline
\end{tabular}

RAGE, receptor of advanced glycation end product.

lymphocyte apoptosis was observed. An alternative model for late hypoimmune events involves CLP in mice and subsequent removal of the necrotic cecum, which can lead to a complete recovery. Peritoneal macrophages isolated from CLP mice that had cecectomy four days after sepsis induction had lower IL-6 production, indicating a hypoimmune state (81). IFN- $\gamma$ production by splenocytes was suppressed in CLP mice but was reversed by cecectomy and IL-10 injection (82). Recently, serum cytokine analysis in a community-acquired pneumonia cohort showed that mortality was not predicted by either proinflammatory IL- 6 or antiinflammatory IL-10, but high levels of both cytokines were more predictive. Thus, by the time of hospital admission, high levels of IL- 6 and IL-10 portend the worst mortality; either the early proinflammatory phase had passed or the early and late phase are coincident (83), which requires further investigation.

Additional clinically relevant factors. Other issues in model development to be considered include, first, that host susceptibility to pathogenic factors is species dependent. For instance, rodents are much less sensitive to LPS than humans (67). Second, bacterial virulence factors such as microbial toxins can accelerate sepsis. Alverdy and colleagues (84) performed a 30\% hepatectomy, which allowed exotoxin A to be disseminated systematically after direct injection of $P$. aeruginosa into the cecum. The mortality rate of this sepsis model could be increased to $100 \%$ by modifying the virulence of bacteria.

Large animal models and bacterial infusion/inoculation

Large animal sepsis models have been developed using LPS infusion, CLP surgery, and bacterial infusion and inoculation. Since these larger animals can be fully instrumented for measurement of circulatory parameters and continuous infusion of fluids and therapeutic agents, ICU fluid management protocols can be simulated, and hemodynamic status can be documented. Fink et al. implanted a fibrin clot containing live $E$. coli in the peritoneal cavity of dogs, which increased cardiac output and decreased blood pressure and systemic vascular resistance (SVR) (85). This canine sepsis model has been used for drug evaluation (86-89). Recently a new canine sepsis model induced by intrabronchial Staphylococcus aureus administration has been developed (90). In this model, treatment with mechanical ventilation, antibiotics, fluids, vasopressors, sedatives, and analgesics was adjusted based on algorithms similar to the care provided for human sepsis. Renal dysfunction evaluated by serum creatinine and BUN was found in acute non-survivors ( $<24$ hours) and decreased urine output in subacute non-survivors (24-96 hours). In a baboon model, a hyperdynamic state is preactivated by injection of killed bacteria followed by injection with live E. coli; renal dysfunction was also demonstrated (91-93). It is of note that renal histological changes, including tubular epithelial cell injury, fibrin deposits, and inflammatory cell infiltration, were found in this model.

Hemodynamics. Failure of the renal circulation is thought to be a crucial factor for developing sepsis-induced AKI; despite lack of demonstrated efficacy, low-dose dopamine is often administered to preserve renal blood flow (RBF). However, in recent reports with a large animal sepsis model, AKI developed even with increased RBF. In sheep infused continuously with live E. coli, serum creatinine increased despite a more than doubled $\operatorname{RBF}(94,95)$; notably, the expected redistribution of intrarenal circulation between cortex and medulla (measured by implanted laser Doppler flow probes) was also absent (96). On the other hand, rodent sepsis models of 
Table 4

Characteristics of TLRs

\begin{tabular}{|c|c|c|c|}
\hline & Ligand(s) & Location & Adapter(s) \\
\hline TLR1 & Triacyl lipopeptides & Cell surface & MyD88/MAL \\
\hline TLR2 & $\begin{array}{l}\text { Glycolipids, lipopeptides, } \\
\text { lipoproteins, zymosan, } \\
\text { lipoteichoic acid, } \\
\text { HSP70, HMGB1? }\end{array}$ & Cell surface & MyD88/MAL \\
\hline TLR3 & dsRNA & Lysosome/endosome & TRIF \\
\hline TLR4 & $\begin{array}{l}\text { LPS, fibrinogen, heparan } \\
\text { sulfate, hyaluronic acid, } \\
\text { HSPs, HMGB1? }\end{array}$ & Cell surface & $\begin{array}{c}\text { MyD88/MAL/ } \\
\text { TRIF/TRAM }\end{array}$ \\
\hline TLR5 & Flagellin & Cell surface & MyD88 \\
\hline TLR6 & Diacyl lipopeptides & Cell surface & MyD88/MAL \\
\hline TLR7 & SSRNA & Lysosome/endosome & MyD88 \\
\hline TLR8 & ssRNA & Lysosome/endosome & MyD88 \\
\hline TLR9 & Unmethylated CpG & Lysosome/endosome & MyD88 \\
\hline TLR10 & Unknown & Cell surface & Unknown \\
\hline
\end{tabular}

signals (111) and perhaps even act as a TLR ligand (112). Myeloid differentiation factor 88 (MyD88) is an adapter protein for all TLRs, except TLR3, linking receptors with downstream kinases (106). These MyD88-dependent pathways induce activation of NF- $\mathrm{KB}$ and cytokine production such as TNF- $\alpha$.

TLR4 and MyD88. TLRs are expressed primarily in immune cells but also in solid organs including brain, heart, lung, liver, and kidney (113-117). TLRs expressed in the kidney play a crucial role in ischemia/reperfusion injury $(118,119)$ but not LPS-induced injury (26). In a study with renal cross-transplantation between wild-type mice and mice from the $\mathrm{C} 3 \mathrm{H} / \mathrm{HeJ}$ strain, which lacks TLR4 function due to a missense mutation, the authors concluded that nonrenal TLR4 plays a major role, but renal TLR4 plays only a minor role in LPS-induced, TNF- $\alpha$-mediated renal injury consisting of tubular and vascular apoptosis.

LPS infusion or CLP developed renal microcirculatory failure, as evaluated by more precise methods, including intravital two-photon video microscopy $(13,48,97,98)$. It should be noted that these rodent sepsis models might have insufficient fluid resuscitation. According to an alternative, inadequately tested hypothesis, AKI is a successful adaptation that protects the body from losing sodium by using tubuloglomerular feedback (TGF) to reduce GFR (99). In septic humans, microcirculatory derangements can be detected by orthogonal polarization spectral imaging (100). Indeed, mortality was better predicted by microcirculatory failure than systemic hemodynamics $(101,102)$. Further investigation is necessary to clarify the relative contribution of global RBF and microcirculation to sepsis and sepsis-induced AKI and whether defects in one of these vessel beds can be treated without compromising the other.

\section{Potential targets for intervention}

We summarize here a few of the many recently emerging potential therapeutic targets for intervention in sepsis and sepsis-induced AKI (Table 3). Developers of potential therapeutic targets still face a daunting set of conceptual and logistical hurdles to improve upon the current translational success rate. Ideally, they should be evaluated in several different clinically relevant sepsis animal models to minimize failures of translation to clinical trials. Timing should also be considered: most therapeutic candidates are administered to animal models before or at the time of sepsis initiation, but they should also be tested for their limits of efficacy after delayed treatment, i.e., their window of therapeutic opportunity. For instance, treatment by ethyl pyruvate or antibodies against high-mobility group box 1 (HMGB1) improved sepsis when administered as late as 24 hours after onset $(34,103-105)$. However, these time frames would be difficult to directly translate to clinical trials without mechanism-based surrogate biomarkers, considering that animal models diverge more at later times.

\section{Toll-like receptors: bitting the snooze button}

Inflammation in sepsis is largely initiated by TLRs, which detect not only a wide range of microbial diversity, but also cellular constituents released due to tissue injury, triggering innate immune responses (106-110) (Table 4). HMGB1 can potentiate TLR ligand
However, because only BUN was used to evaluate renal function, prerenal azotemia (from systemic TLR4-mediated inflammation) cannot be ruled out, which would support the opposite conclusion (26). TLR4 was induced in proximal and distal tubules after CLP, perhaps as a protective response to endogenous TLR4 ligands and/or anticipated endotoxin, and post-CLP injection of LPS was taken up by proximal tubular cells (120). Direct LPS-induced renal tubular cell damage might partly explain why many antiinflammatory treatments failed to prevent renal injury. It is reported that polymicrobial sepsis in rodents or humans is not dependent on TLR4 (50, 121-123). Sepsis-induced AKI and other organ injury were compared in wild-type mice, TLR4-deficient $\mathrm{C} 3 \mathrm{H} / \mathrm{HeJ}$ mice, and MyD88-knockout mice (50). While sepsis-induced AKI was dependent on MyD88 but not on TLR4, liver injury was independent of either MyD88 or TLR4. On the other hand, blockade of TLR4 pathway in a single bacteria (E. coli) injection model showed remarkable protection (124), demonstrating that TLR4 inhibition can be highly effective when given early, despite the presence of other TLR ligands, and suggests that the uniformity of a bolus injection of a single pathogen may be more amenable to treatment than a sustained polymicrobial infection. Moreover, genetic disruption of MyD88 in mice improved survival rate in a CASP model but not in a standard CLP model $(122,125)$. Survival analysis was not performed in our CLP model in aged, fluid- and antibiotictreated mice (50). These disparate results highlight the importance of systematically analyzing differences among sepsis animal models. It is of note that humans lacking MyD88 activity experience only a limited set of severe infections; they are susceptible to streptococcal and staphylococcal bacteria infection but typically resistant to other microbes (S126). As described above, TLR4 pathway blockade was effective in a mouse sepsis model of E. coli injection. These data indicate that TLRs may play different roles in different types of sepsis.

TLR9. The TLR9 pathway is involved in antiviral immune responses but also recognizes bacterial DNA and specific unmethylated CPG-containing synthetic immunostimulatory oligonucleotides (ODNs) (S127). TLR9 deficiency or an ODN inhibitor of TLR9 improved CLP sepsis-induced mortality and AKI, with improvements in downstream systemic pro- and antiinflamma- 
tory cytokines, bacteria clearance, and splenic apoptosis in both standard (young, untreated) and aged fluid/antibiotic-treated mouse CLP models (ref. 42, S128). TLR9 is primarily expressed on dendritic cells, which can play a protective role in CLP-induced sepsis (S129). The antimalarial drug chloroquine, which inhibits the TLR9 pathway in vitro by preventing lysosomal acidification (S130), also improves sepsis with a 6-hour therapeutic window (ref. 42, S131). A TLR9-selective inhibitor, iCpG, was still effective 12 hours after CLP (S128), opening up the possibility that TLR9 is not limited to simple detection of bacterial CpG and could be involved in amplification or resolution of inflammation. However, in some other infection models, such as meningococcal sepsis and Gram-negative bacterial pneumonia, blocking the TLR9 pathway was harmful (S132, S133), whereas it was beneficial in the CLP model (42). These discrepancies may be pathogen specific or severity specific (i.e., the intensity of infection alters the expression and timing of the target).

\section{Preventing end-organ damage: softening the blow}

To prevent end-organ damage by sepsis, it will be important not only to control dysregulated immunological reactions against infection but also to reduce subsequent activation of nonimmunological responses that damage organs. Many experimental therapies are highly effective when given early - either prior to the septic insult or shortly thereafter; however, they lose effectiveness if given after the animal becomes clinically sick, corresponding to the typical delay in diagnosis of sepsis. This is especially true with antiinflammatory therapies, where a therapeutic target may only be active transiently. While we do not often have the luxury of such early treatment in patients as we do with animal models, end-organ failure occurs well after diagnosis, which provides an opportunity to intercept these late signals and mediators to minimize irreversible organ damage. If organ failure can be decreased, it should have a significant impact on long-term outcomes for patients who survive sepsis.

$V E G F$. VEGF promotes microvascular integrity (S134), but high levels of VEGF can cause vascular leakage by destruction of vascular barrier function (S135). In human sepsis, plasma VEGF levels were increased, and VEGF concentration at admission correlated with the severity of multiple organ dysfunction (S136, S137). By trapping circulating VEGF, soluble FLT1 peptide improved survival of a mouse CLP model, even when administered 24 hours after sepsis induction (S138, S139). In the mouse two-stage model of CKD-sepsis described above, plasma VEGF levels in CKD animals were higher than normal and significantly increased by sepsis. VEGF is cleared by the kidneys, which could explain why CKD enhances sepsis (78).

C5a. Extensive studies point to the complement system, particularly C5a, as a therapeutic target (reviewed in ref. S140). Recently, Rittirsch et al. reported that a C5a receptor, C5L2, which was previously considered a decoy receptor for C5a, contributes to the development of sepsis by inducing HMGB1 release from macrophages (S141). Anti-C5a antibody treatment improved CLP-induced renal pathological injury (S142).

\section{The neuroimmune axis: keeping a clear head}

Stress, from both a neuroendocrine and cellular point of view, is an added dimension that complicates the ability of the host to fight infections. Much has been written about glucocorticoids as antiinflammatory agents (S143, S144) and catecholamines as pressors, but usually the central nervous system is an afterthought in terms of pathophysiology or considered too complex to study.

Increasing parasympathetic outflow. Vagus nerve stimulation attenuates an inflammatory response induced by LPS, including TNF- $\alpha$ release from activated macrophages (S145). This cholinergic antiinflammatory pathway is thought to act through the nicotinic acetylcholine receptor $\alpha 7$ subunit ( $\alpha 7 \mathrm{nAChR})$ (S146). A simple, direct mechanism may be involved, as acetylcholine inhibits HMGB1 release from isolated macrophages through $\alpha 7 \mathrm{nAchR}$ (S147). In vivo, treatment with nicotine decreases serum HMGB1 levels and improves survival of LPS- and CLP-induced sepsis animal models (S147). Splenectomy and selective abdominal vagotomy diminish the antiinflammatory effects (TNF- $\alpha$ and HMGB1) of vagus nerve stimulation or nicotine treatment in LPS and CLP sepsis mice (S148). In splenectomized mice, nicotine treatment not only fails to improve sepsis, but it worsens survival and increases serum HMGB1 levels. These divergent results illustrate that interaction between central nervous system and innate immune defense system is not straightforward. In addition to the parasympathetic nervous system, the sympathetic nervous system is also thought to play a role in sepsis (reviewed in ref. S149).

Pituitary hormones. Several neuroendocrine mediators have recently been revealed to have immunomodulatory actions (reviewed in ref. S150). We describe two recently reported examples. $\alpha$-Melanocyte-stimulating hormone $(\alpha-\mathrm{MSH})$ is an antiinflammatory proopiomelanocortin-derived (POMC-derived) peptide from pituitary gland, with additional effects including regulation of food intake and energy expenditure (S151, S152). POMC levels are decreased in septic rats (S153) and patients (S154). $\alpha$-MSH and its analog attenuate sepsis-induced AKI and mortality in the mouse CLP model by improving systemic hemodynamics, pro- and antiinflammatory actions, renal and splenic NF- $\mathrm{KB}$ activation, and splenocyte apoptosis (ref. 64, S155). Ghrelin is a gastrointestinal tract-derived orexigenic peptide with potent antiinflammatory properties (S156, S157). Ghrelin improved sepsis induced by LPS injection or CLP up to 24 hours later by decreasing HMGB1 secretion from macrophages (S158). Further, some of the beneficial effects of ghrelin were sensitive to vagotomy (S159). Therefore, a thematic framework appears to be coalescing around HMGB1 as an important nexus between the immune response and the sympathetic, parasympathetic, and hypothalamic-pituitary-adrenal axes.

\section{Cellular therapies: fighting smarter}

Conventional therapeutic strategies are systemic in nature, but many symptoms and complications of sepsis emanate from a local site of initial infection. Rather than applying a uniform treatment globally, cellular therapies seek to enhance responses to local conditions. Cellular therapies cannot replace antibiotics, but serve as an adjunct to fight pockets of infection or regulate inflammation on a local scale.

Neutrophil extracellular traps. After bacteria bind to platelet TLR4, neutrophils are activated, resulting in the production of extracellular fibers called neutrophil extracellular traps (NETs) composed of granule proteins and chromatin that also kill bacteria in blood and tissue (S160-S162). NET formation may also injure endothelium and tissue, as neutrophil or platelet depletion attenuated endothelial and hepatic injury in an LPS-induced sepsis model (S161). Depletion of platelets also reduced neutrophil recruitment to the lung and lung edema in CLP-induced sepsis (S163). Because of its dependence on TLR4, NET formation may account for some 
Table 5

Biomarker candidates for sepsis-induced AKI

\begin{tabular}{lccc} 
Candidate & Mechanistic axis & Tested in humans & Ref. \\
NGAL & Neutrophil/inflammation & Pediatric sepsis AKI & S192, S193 \\
KIM-1 & Proximal tubule damage & Adult AKI including sepsis & S194 \\
L-FABP & Proximal tubule damage & Adult sepsis AKI & S195 \\
IL-18 & Inflammation & Pediatric sepsis AKI & S196 \\
Exosomal ATF3 & Tubule transcription factor & Adult sepsis AKI & S197 \\
Meprin-1- $\alpha$ & Proximal tubule damage & No & 44 \\
Cyclophilin A & Liver damage & No & S198 \\
\hline
\end{tabular}

NGAL, neutrophil gelatinase-associated lipocalin; L-FABP, L-type fatty acid-binding protein; ATF3, activating transcription factor 3 .

differences in AKI found in LPS versus CLP models; however, the effect may be indirect, as NETs were found in liver sinusoids and pulmonary capillaries but not in the kidney (S161).

Mesenchymal stem cells/bone marrow stromal cells. Mesenchymal stem cells (MSCs) have been proposed to have immunosuppressive properties and reduce inflammation (S164, S165) by inhibiting T cell proliferation and B cell function (S166). MSC treatment improved severe acute human graft-versus-host disease (S167) and LPS-induced acute lung injury (S168). MSCs reduced nonseptic AKI through paracrine effects (S169, S170). Recently, we demonstrated that MSCs reduced mortality and multiple organ failure in a mouse CLP model (S171). MSCs transiently moved near monocytes/macrophages and reprogrammed them to produce the antiinflammatory cytokine IL-10 via $\mathrm{PGE}_{2}$ signaling. MSCs from adipose tissue were also effective in treating CLP-induced sepsis (S172). Because allogeneic MSCs can be injected intravenously into the host without any immunosuppression treatment and can be expanded ex vivo, MSC therapy has great potential for human sepsis. Systemic $\mathrm{PGE}_{2}$ administration has not been demonstrated to treat sepsis, whereas MSCs can respond to changing conditions to deliver a localized, transient, proportionate release of $\mathrm{PGE}_{2}$. This smarter local delivery vehicle should be effective in a broader range of patients and reduce side effects including immunosuppression.

\section{New biomarkers in sepsis-induced AKI}

Despite numerous sophisticated scoring systems (acute physiology and chronic health evaluation [APACHE; ref. S173], sepsisrelated organ failure assessment [SOFA, ref. S174], etc.), organ failure metrics (e.g., ventilator-free days, dialysis-free days, etc.), and pro- and antiinflammatory biomarkers (e.g., IL-6, procalcitonin, triggering receptor expressed on myeloid cells-1 [TREM-1], IL-10, etc.) mortality is still used as the gold standard for evaluating new therapeutic agents and strategies. Because mortality from sepsis is increased dramatically when complicated by AKI, early detection and accurate evaluation of AKI is important in septic patients. AKI has been mainly diagnosed by serum creatinine concentration, which can partly reflect GFR as a function of solute elimination by the kidney. A recent international consensus definition for AKI attempts to adjust for large increases in risk that accompany small increases in serum creatinine (6). However, this definition still depends on changes in serum creatinine concentration and urine output. Unfortunately, serum creatinine does not accurately reflect the GFR in critically ill patients with fluid shifts who are not in steady state. We recently discovered that sepsis also decreases creatinine production (S175). In bilaterally nephrectomized (BNx) mice, sepsis reduced serum creatinine but increased nonrenal organ injury markers and serum cytokine levels, as expected. Treatment of sepsis with chloroquine decreased nonrenal organ injury markers but paradoxically increased serum creatinine. These data suggest that evaluation of kidney injury by serum creatinine alone would severely underestimate renal injury, a serious failure of early diagnosis of sepsis-induced AKI, and lead to an incorrect assessment of drug efficacy.

One approach to finding a better alternative to serum creatinine is to test new renal biomarkers that were initially developed to detect other forms of AKI (S176). A few studies of novel renal biomarkers have been reported in patients with sepsis-induced AKI (reviewed in refs. S177, S178 and summarized in Table 5).

The development of drug-biomarker pairs has been critical in other areas of medicine, especially oncology. Many agents, such as TNF- $\alpha$, can have opposing effects on sepsis in different contexts (ref. 41, S179). Therefore, it will be critical to match agents with a particular clinical context or "niche": agent(s) must be given in the correctly defined subpopulation that may be susceptible to the agent, as in the case of the anticancer drugs Gleevec and Herceptin (S180). Biomarkers can also be used to determine when the drug target is available and active; how to adjust the dose to saturate the target; and when to stop due to the recovery from disease or no expected response. Giving agents to all the septic patients without any stratification by biomarkers has been unsuccessful at best, and in many cases harmful (S144, S181, S182). Considering the complexity of sepsis within the patient population, as well as the dynamic nature of sepsis in an individual patient, a panel of biomarkers may be needed, based on each available therapy. For example, patients can be screened for low endogenous protein C before administration of activated protein C (S183). The use of a genetically heterogeneous animal model may be advantageous over the use of inbred strain-based models for development of biomarkers and biomarker-therapeutic pairs.

\section{Conclusions}

Lessons learned. The failure to translate benefits seen in animal models to the clinical setting has caused much soul searching in the sepsis community (14-16). In the last several decades, animal modelers have focused primarily on replicating clinical stages (hemodynamic, inflammatory, and, most recently, immunosuppression) and outcomes (organ failure, mortality rate). We have incorporated results from human epidemiological studies to design and validate our animal models. We found that replicating genetic heterogeneity and critical clinical risk factors such as advanced age and comorbid conditions (e.g., CKD) led to improved models of sepsis and sepsis-induced AKI. Such disease models have given the field new ways to addresses the vexing problem of how to mimic a complex human disease in animal models. In the LPS or simple CLP models without the genetic diversity or risk factors (age, underlying disease), we had great difficulty titrating the dose of LPS or CLP to produce AKI; a mild insult did not cause AKI, but a slightly stronger insult caused death without AKI. Addition of genetic diversity or risk factors made the animals more susceptible to sepsis. When the severity of insult is then decreased, these mod- 
els became less pathogen dependent and more host dependent, allowing the development of multiple organ failure to unfold during the course of sepsis.

The added risk factors enabled a failed human clinical trial to be replicated in an animal model of disease (48), an often overlooked criterion for validating animal models. For any animal model of sepsis, it will be important to demonstrate that the model possesses a "negative predictive value," i.e., if a therapeutic lacks efficacy in the treatment of human disease, does the animal model similarly predict therapeutic failure? Addition of risk factors also changed the responsiveness to treatments of sepsis. Monotherapies effective in a simpler CLP model were no longer effective in more complex models; combination therapies were needed to prevent disease in the more complex models $(63,78)$. Perhaps similar drug combinations will be required for human sepsis, analogous to combination chemotherapy for cancer (S184) or HIV (S185).

Future directions for animal models. The disease models to date have successfully mimicked the initial stages of sepsis; however, the later stages of immunosuppression and secondary infections have only been addressed by a few studies, and many mechanistic questions have yet to be answered. When comparing different treatment windows of opportunity in different animal models, some normalizing principle may be necessary, such as initiation of treatment relative to mean survival time.

Can animal models help lead us to develop new therapeutic strategies and diagnostic tools? Although there has been some success with early goal-directed therapy (S186), this preemptive strategy has a short/narrow window of opportunity, probably because the hemodynamically amenable phase is short lived, then patients diverge into multiple mechanistic directions. Because patients with sepsis are exceedingly heterogeneous, with individual "disease clocks," we need to improve our ability to monitor and ultimately predict how patients move along their individual disease trajectory (S187).

Furthermore, we are accustomed to having a single fixed therapeutic target (e.g., HMG-CoA reductase for hypercholesterolemia) (S188), but in sepsis, the therapeutic targets vary with the underlying preexisting conditions, etiology of sepsis, time course, and disease trajectory. Subsets of the septic patient population, such as those with meningococcemia, can be replicated individually in animal models. However, cases are typically more complex, and we need new classification schemes and biomarkers to identify which informative subset a patient belongs to, how far along the disease trajectory a patient has progressed, and which biological targets are active at any given time. Ideally, drug target assays would be developed that indicate whether an administered drug was hitting and saturating the target, allowing proper dose titration and anticipation of side effects. Such schemes might even allow for the selection of a rationally designed, synergistic combination therapy. The predictive accuracy of such schemes can then be used to design more accurate animal models in an interactive, iterative process.

Note: References S126-S198 are available online with this article; doi:10.1172/JCI39421DS1.

\section{Acknowledgments}

This research was supported by the Intramural Research Program of the NIH, NIDDK. We apologize to all the authors whose work we could not cover due to space limitations.

Address correspondence to: Peter S.T. Yuen, Renal Diagnostics and Therapeutics Unit, NIDDK, NIH, 10 Center Drive, Room 3N108, Bethesda, Maryland 20892-1268, USA. Phone: (301) 402-6702; Fax: (301) 402-0014; E-mail: py@nih.gov.
1. Levy, M.M., et al. 2003.2001 SCCM/ESICM/ACCP/ ATS/SIS International Sepsis Definitions Conference. Crit. Care Med. 31:1250-1256.

2. Dellinger, R.P., et al. 2008. Surviving Sepsis Campaign: international guidelines for management of severe sepsis and septic shock: 2008. Crit. Care Med. 36:296-327.

3. Angus, D.C., et al. 2001. Epidemiology of severe sepsis in the United States: analysis of incidence, outcome, and associated costs of care. Crit. Care Med. 29:1303-1310.

4. Martin, G.S., Mannino, D.M., Eaton, S., and Moss, M. 2003. The epidemiology of sepsis in the United States from 1979 through 2000. N. Engl. J. Med. 348:1546-1554.

5. Dombrovskiy, V.Y., Martin, A.A., Sunderram, J., and Paz, H.L. 2007. Rapid increase in hospitalization and mortality rates for severe sepsis in the United States: a trend analysis from 1993 to 2003. Crit. Care Med. 35:1244-1250.

6. Mehta, R.L., et al. 2007. Acute Kidney Injury Network: report of an initiative to improve outcomes in acute kidney injury. Crit. Care. 11:R31.

7. Russell, J.A., et al. 2000. Changing pattern of organ dysfunction in early human sepsis is related to mortality. Crit. Care Med. 28:3405-3411.

8. Uchino, S., et al. 2005. Acute renal failure in critically ill patients: a multinational, multicenter study. JAMA. 294:813-818.

9. Bagshaw, S.M., et al. 2005. Prognosis for long-term survival and renal recovery in critically ill patients with severe acute renal failure: a population-based study. Crit. Care. 9:R700-R709.

10. Neveu, H., Kleinknecht, D., Brivet, F., Loirat, P., and Landais, P. 1996. Prognostic factors in acute renal failure due to sepsis. Results of a prospective multicentre study. The French Study Group on Acute Renal Failure. Nephrol. Dial. Transplant. 11:293-299.

11. Silvester, W., Bellomo, R., and Cole, L. 2001. Epidemiology, management, and outcome of severe acute renal failure of critical illness in Australia. Crit. Care Med. 29:1910-1915.

12. Schrier, R.W., and Wang, W. 2004. Acute renal failure and sepsis. N. Engl. J. Med. 351:159-169.

13. Wu, L., Gokden, N., and Mayeux, P.R. 2007. Evidence for the role of reactive nitrogen species in polymicrobial sepsis-induced renal peritubular capillary dysfunction and tubular injury. J. Am. Soc. Nephrol. 18:1807-1815.

14. Riedemann, N.C., Guo, R.F., and Ward, P.A. 2003. The enigma of sepsis. J. Clin. Invest. 112:460-467.

15. Rittirsch, D., Hoesel, L.M., and Ward, P.A. 2007. The disconnect between animal models of sepsis and human sepsis. J. Leukoc. Biol. 81:137-143.

16. Dyson, A., and Singer, M. 2009. Animal models of sepsis: why does preclinical efficacy fail to translate to the clinical setting? Crit. Care Med. 37:S30-S37.

17. Esmon, C.T. 2004. Why do animal models (sometimes) fail to mimic human sepsis? Crit. Care Med. 32:S219-S222.

18. Hotchkiss, R.S., and Karl, I.E. 2003. The pathophysiology and treatment of sepsis. N. Engl. J. Med. 348:138-150.

19. Riedemann, N.C., Guo, R.F., and Ward, P.A. 2003. Novel strategies for the treatment of sepsis. Nat. Med. 9:517-524.

20. Wichterman, K.A., Baue, A.E., and Chaudry, I.H. 1980. Sepsis and septic shock--a review of laboratory models and a proposal. J. Surg. Res. 29:189-201.

21. Remick, D.G., Newcomb, D.E., Bolgos, G.L., and Call, D.R. 2000. Comparison of the mortality and inflammatory response of two models of sepsis: lipopolysaccharide vs. cecal ligation and puncture.
Shock. 13:110-116.

22. Michie, H.R., et al. 1988. Detection of circulating tumor necrosis factor after endotoxin administration. N. Engl. J. Med. 318:1481-1486.

23. Tracey, K.J., et al. 1987. Anti-cachectin/TNF monoclonal antibodies prevent septic shock during lethal bacteraemia. Nature. 330:662-664.

24. McNamara, M.J., Norton, J.A., Nauta, R.J., and Alexander, H.R. 1993. Interleukin-1 receptor antibody (IL-1 rab) protection and treatment against lethal endotoxemia in mice. J. Surg. Res. 54:316-321.

25. Taveira da Silva, A.M., et al. 1993. Brief report: shock and multiple-organ dysfunction after selfadministration of Salmonella endotoxin. N. Engl. J. Med. 328:1457-1460.

26. Cunningham, P.N., Wang, Y., Guo, R., He, G., and Quigg, R.J. 2004. Role of Toll-like receptor 4 in endotoxin-induced acute renal failure. J. Immunol. 172:2629-2635.

27. Tiwari, M.M., Brock, R.W., Megyesi, J.K., Kaushal, G.P., and Mayeux, P.R. 2005. Disruption of renal peritubular blood flow in lipopolysaccharideinduced renal failure: role of nitric oxide and caspases. Am. J. Physiol. Renal Physiol. 289:F1324-1332.

28. Knotek, M., et al. 2001. Endotoxemic renal failure in mice: role of tumor necrosis factor independent of inducible nitric oxide synthase. Kidney Int. 59:2243-2249.

29. Fisher, C.J., Jr., et al. 1996. Treatment of septic shock with the tumor necrosis factor receptor:Fc fusion protein. N. Engl. J. Med. $334: 1697-1702$.

30. Fisher, C.J., Jr., et al. 1994. Recombinant human interleukin 1 receptor antagonist in the treatment of patients with sepsis syndrome. Results from a randomized, double-blind, placebo-controlled trial. JAMA. 271 :1836-1843.

31. Eskandari, M.K., et al. 1992. Anti-tumor necrosis 
factor antibody therapy fails to prevent lethality after cecal ligation and puncture or endotoxemia. J. Immunol. 148:2724-2730.

32. Brandtzaeg, P., et al. 1988. Systemic meningococcal disease: a model infection to study acute endotoxinemia in man. Prog. Clin. Biol. Res. 272:263-271.

33. Johannes, T., Mik, E.G., and Ince, C. 2009. Nonresuscitated endotoxemia induces microcirculatory hypoxic areas in the renal cortex in the rat. Shock. 31:97-103.

34. Miyaji, T., et al. 2003. Ethyl pyruvate decreases sepsis-induced acute renal failure and multiple organ damage in aged mice. Kidney Int. 64:1620-1631.

35. Wang, W., et al. 2002. Protective effect of renal denervation on normotensive endotoxemiainduced acute renal failure in mice. Am. J. Physiol. Renal Physiol. 283:F583-F587.

36. Wang, W., et al. 2003. Interaction among nitric oxide, reactive oxygen species, and antioxidants during endotoxemia-related acute renal failure. Am. J. Physiol. Renal Physiol. 284:F532-F537.

37. Buras, J.A., Holzmann, B., and Sitkovsky, M. 2005. Animal models of sepsis: setting the stage. Nat. Rev Drug Discov. 4:854-865.

38. Deitch, E.A. 2005. Rodent models of intra-abdominal infection. Shock. 24(Suppl. 1):19-23.

39. Rittirsch, D., Huber-Lang, M.S., Flierl, M.A., and Ward, P.A. 2009. Immunodesign of experimental sepsis by cecal ligation and puncture. Nat. Protoc. 4:31-36.

40. Freise, H., Bruckner, U.B., and Spiegel, H.U. 2001. Animal models of sepsis. J. Invest. Surg. 14:195-212.

41. Alexander, H.R., et al. 1991. Treatment with recombinant human tumor necrosis factor-alpha protects rats against the lethality, hypotension, and hypothermia of gram-negative sepsis. J. Clin. Invest. 88:34-39.

42. Yasuda, H., et al. 2008. Chloroquine and inhibition of Toll-like receptor 9 protect from sepsis-induced acute kidney injury. Am. J. Physiol. Renal Physiol. 294:F1050-F1058

43. Ertel, W., et al. 1991. The complex pattern of cytokines in sepsis. Association between prostaglandins, cachectin, and interleukins. Ann. Surg. 214:141-148.

44. Holly, M.K., et al. 2006. Biomarker and drug-target discovery using proteomics in a new rat model of sepsis-induced acute renal failure. Kidney Int. 70:496-506.

45. Ganopolsky, J.G., and Castellino, F.J. 2004. A protein $\mathrm{C}$ deficiency exacerbates inflammatory and hypotensive responses in mice during polymicrobial sepsis in a cecal ligation and puncture model. Am. J. Pathol. 165:1433-1446.

46. Hollenberg, S.M., et al. 2001. Characterization of a hyperdynamic murine model of resuscitated sepsis using echocardiography. Am. J. Respir. Crit. Care Med. 164:891-895.

47. Echtenacher, B., Falk, W., Mannel, D.N., and Krammer, P.H. 1990. Requirement of endogenous tumor necrosis factor/cachectin for recovery from experimental peritonitis. J. Immunol. 145:3762-3766.

48. Yasuda, H., Yuen, P.S., Hu, X., Zhou, H., and Star, R.A. 2006. Simvastatin improves sepsis-induced mortality and acute kidney injury via renal vascular effects. Kidney Int. 69:1535-1542.

49. Ayala, A., and Chaudry, I.H. 1996. Immune dysfunction in murine polymicrobial sepsis: mediators, macrophages, lymphocytes and apoptosis. Shock. 6(Suppl. 1):S27-S38.

50. Dear, J.W., et al. 2006. Sepsis-induced organ failure is mediated by different pathways in the kidney and liver: acute renal failure is dependent on MyD88 but not renal cell apoptosis. Kidney Int. 69:832-836.

51. Hoesel, L.M., et al. 2005. Harmful and protective roles of neutrophils in sepsis. Shock. 24:40-47.

52. Haybron, D.M., et al. 1987. Alterations in renal perfusion and renal energy charge in murine peri- tonitis. Arch. Surg. 122:328-331.

53. Kalechman, Y., et al. 2002. Anti-IL-10 therapeutic strategy using the immunomodulator AS101 in protecting mice from sepsis-induced death: dependence on timing of immunomodulating intervention. J. Immunol. 169:384-392.

54. Matsukawa, A., Kaplan, M.H., Hogaboam, C.M., Lukacs, N.W., and Kunkel, S.L. 2001. Pivotal role of signal transducer and activator of transcription (Stat) 4 and Stat 6 in the innate immune response during sepsis. J. Exp. Med. 193:679-688.

55. Yang, S., and Hauptman, J.G. 1994. The efficacy of heparin and antithrombin III in fluid-resuscitated cecal ligation and puncture. Shock. 2:433-437.

56. Kuhlmann, M.K., et al. 1994. New experimental model of acute renal failure and sepsis in rats. JPEN J. Parenter. Enteral. Nutr. 18:477-485.

57. Pedersen, P.V., et al. 1989. Hemodynamic and metabolic alterations during experimental sepsis in young and adult rats. Surg. Gynecol. Obstet. 168:148-156

58. Muenzer, J.T., et al. 2006. Pneumonia after cecal ligation and puncture: a clinically relevant "twohit" model of sepsis. Shock. 26:565-570.

59. Neild, G.H. 2001. Multi-organ renal failure in the elderly. Int. Urol. Nephrol. 32:559-565.

60. Saito, H., Sherwood, E.R., Varma, T.K., and Evers, B.M. 2003. Effects of aging on mortality, hypothermia, and cytokine induction in mice with endotoxemia or sepsis. Mech. Ageing Dev. 124:1047-1058.

61. Turnbull, I.R., et al. 2003. Effects of age on mortality and antibiotic efficacy in cecal ligation and puncture. Shock. 19:310-313.

62. Dear, J.W., et al. 2005. Dendrimer-enhanced MRI as a diagnostic and prognostic biomarker of sepsisinduced acute renal failure in aged mice. Kidney Int. 67:2159-2167.

63. Leelahavanichkul, A., et al. 2008. Methyl-2-acetamidoacrylate, an ethyl pyruvate analog, decreases sepsis-induced acute kidney injury in mice. Am. J. Physiol. Renal Physiol. 295:F1825-F1835.

64. Doi, K., et al. 2008. AP214, an analogue of alphamelanocyte-stimulating hormone, ameliorates sepsis-induced acute kidney injury and mortality. Kidney Int. 73:1266-1274.

65. Langenberg, C., Bagshaw, S.M., May, C.N., and Bellomo, R. 2008. The histopathology of septic acute kidney injury: a systematic review. Crit. Care. 12:R38.

66. Sato, T., Kamiyama, Y., Jones, R.T., Cowley, R.A., and Trump, B.F. 1978. Ultrastructural study on kidney cell injury following various types of shock in 26 immediate autopsy patients. Adv. Shock Res. 1:55-69.

67. Liptak, P., and Ivanyi, B. 2006. Primer: histopathology of calcineurin-inhibitor toxicity in renal allografts. Nat. Clin. Pract. Nephrol. 2:398-404.

68. Wan, L., et al. 2008. Pathophysiology of septic acute kidney injury: what do we really know? Crit. Care Med. 36:S198-S203.

69. Chawla, L.S., et al. 2007. Elevated plasma concentrations of IL-6 and elevated APACHE II score predict acute kidney injury in patients with severe sepsis. Clin. J. Am. Soc. Nephrol. 2:22-30.

70. Doetschman, T. 1999. Interpretation of phenotype in genetically engineered mice. Lab. Anim. Sci. 49:137-143.

71. Williams, S.M., Haines, J.L., and Moore, J.H. 2004. The use of animal models in the study of complex disease: all else is never equal or why do so many human studies fail to replicate animal findings? Bioessays. 26:170-179.

72. Guidet, B., Aegerter, P., Gauzit, R., Meshaka, P., and Dreyfuss, D. 2005. Incidence and impact of organ dysfunctions associated with sepsis. Chest. 127:942-951

73. Mehta, R.L., et al. 2004. Spectrum of acute renal failure in the intensive care unit: the PICARD experience. Kidney Int. 66:1613-1621.
74. Naqvi, S.B., and Collins, A.J. 2006. Infectious complications in chronic kidney disease. Adv. Chronic Kidney Dis.13:199-204.

75. Sarnak, M.J., and Jaber, B.L. 2000. Mortality caused by sepsis in patients with end-stage renal disease compared with the general population. Kidney Int. 58:1758-1764.

76. Thamer, M., Ray, N.F., Fehrenbach, S.N., Richard, C., and Kimmel, P.L. 1996. Relative risk and economic consequences of inpatient care among patients with renal failure. J. Am. Soc. Nephrol. 7:751-762.

77. James, M.T., et al. 2008. Risk of bloodstream infection in patients with chronic kidney disease not treated with dialysis. Arch. Intern. Med. 168:2333-2339.

78. Doi, K., et al. 2008. Pre-existing renal disease promotes sepsis-induced acute kidney injury and worsens outcome. Kidney Int. 74:1017-1025.

79. Pugin, J. 2007. Immunostimulation is a rational therapeutic strategy in sepsis. Novartis Found. Symp. 280:21-27; discussion 27-36, 160-164.

80. Steinhauser, M.L., et al. 1999. IL-10 is a major mediator of sepsis-induced impairment in lung antibacterial host defense. J. Immunol. 162:392-399.

81. Xiao, H., Siddiqui, J., and Remick, D.G. 2006. Mechanisms of mortality in early and late sepsis. Infect. Immun. 74:5227-5235.

82. Manley, M.O., O'Riordan, M.A., Levine, A.D., and Latifi, S.Q. 2005. Interleukin 10 extends the effectiveness of standard therapy during late sepsis with serum interleukin 6 levels predicting outcome. Shock. 23:521-526.

83. Kellum, J.A., et al. 2007. Understanding the inflammatory cytokine response in pneumonia and sepsis: results of the Genetic and Inflammatory Markers of Sepsis (GenIMS) Study. Arch. Intern. Med. 167:1655-1663.

84. Laughlin, R.S., et al. 2000. The key role of Psendomonas aeruginosa PA-I lectin on experimental gutderived sepsis. Ann. Surg. 232:133-142.

85. Fink, M.P., MacVittie, T.J., and Casey, L.C. 1984. Inhibition of prostaglandin synthesis restores normal hemodynamics in canine hyperdynamic sepsis. Ann. Surg. 200:619-626.

86. Kalil, A.C., et al. 2006. Preclinical trial of L-arginine monotherapy alone or with $\mathrm{N}$-acetylcysteine in septic shock. Crit. Care Med. 34:2719-2728.

87. Natanson, C., et al. 1990. Antibiotics versus cardiovascular support in a canine model of human septic shock. Am. J. Physiol. 259:H1440-H1447.

88. Quezado, Z.M., et al. 1993. A controlled trial of HA-1A in a canine model of gram-negative septic shock. JAMA. 269:2221-2227.

89. Sevransky, J.E., et al. 1997. Tyrphostin AG 556 improves survival and reduces multiorgan failure in canine Escherichia coli peritonitis. J. Clin. Invest. 99:1966-1973.

90. Minneci, P.C., et al. 2007. A canine model of septic shock: balancing animal welfare and scientific relevance. Am. J. Physiol. Heart Circ. Physiol. 293:H2487-H2500.

91. Carraway, M.S., et al. 2003. Blockade of tissue factor: treatment for organ injury in established sepsis. Am. J. Respir. Crit. Care Med. 167:1200-1209.

92. Welty-Wolf, K.E., et al. 2001. Coagulation blockade prevents sepsis-induced respiratory and renal failure in baboons. Am. J. Respir. Crit. Care Med. 164:1988-1996.

93. Welty-Wolf, K.E., et al. 2006. Blockade of tissue factor-factor $\mathrm{X}$ binding attenuates sepsis-induced respiratory and renal failure. Am. J. Physiol. Lung Cell. Mol. Physiol. 290:L21-L31.

94. Langenberg, C., Wan, L., Egi, M., May, C.N., and Bellomo, R. 2006. Renal blood flow in experimental septic acute renal failure. Kidney Int. 69:1996-2002.

95. Langenberg, C., Wan, L., Egi, M., May, C.N., and Bellomo, R. 2007. Renal blood flow and function during recovery from experimental septic acute 
kidney injury. Intensive Care Med. 33:1614-1618.

96. Di Giantomasso, D., Morimatsu, H., May, C.N., and Bellomo, R. 2003. Intrarenal blood flow distribution in hyperdynamic septic shock: effect of norepinephrine. Crit. Care Med. 31:2509-2513.

97. Wu, L., et al. 2007. Peritubular capillary dysfunction and renal tubular epithelial cell stress following lipopolysaccharide administration in mice. Am. J. Physiol. Renal Physiol. 292:F261-F268.

98. Gupta, A., et al. 2007. Activated protein C ameliorates LPS-induced acute kidney injury and downregulates renal iNOS and angiotensin 2. Am.J. Physiol. Renal Physiol. 293:F245-F254.

99. Thurau, K., and Boylan, J.W. 1976. Acute renal success. The unexpected logic of oliguria in acute renal failure. Am. J. Med. 61:308-315.

100.De Backer, D., Creteur, J., Preiser, J.C., Dubois, M.J., and Vincent, J.L. 2002. Microvascular blood flow is altered in patients with sepsis. Am. J. Respir. Crit. Care Med. 166:98-104.

101. De Backer, D., et al. 2006. The effects of dobutamine on microcirculatory alterations in patients with septic shock are independent of its systemic effects. Crit. Care Med. 34:403-408.

102.Sakr, Y., Dubois, M.J., De Backer, D., Creteur, J., and Vincent, J.L. 2004. Persistent microcirculatory alterations are associated with organ failure and death in patients with septic shock. Crit. Care Med. 32:1825-1831.

103. Yang, H., et al. 2004. Reversing established sepsis with antagonists of endogenous high-mobility group box 1. Proc. Natl. Acad. Sci. U. S. A. 101:296-301.

104.Wang, H., et al. 1999. HMG-1 as a late mediator of endotoxin lethality in mice. Science. 285:248-251.

105. Ulloa, L., et al. 2002. Ethyl pyruvate prevents lethality in mice with established lethal sepsis and sys- temic inflammation. Proc. Natl. Acad. Sci. U. S. A. 99:12351-12356.

106.Akira, S., and Takeda, K. 2004. Toll-like receptor signalling. Nat. Rev. Immunol. 4:499-511.

107. Coussens, L.M., and Werb, Z. 2002. Inflammation and cancer. Nature. 420:860-867.

108. Medzhitov, R. 2007. Recognition of microorganisms and activation of the immune response. Nature. 449:819-826.

109.Seong, S.Y., and Matzinger, P. 2004. Hydrophobicity: an ancient damage-associated molecular pattern that initiates innate immune responses. Nat. Rev. Immunol. 4:469-478.

110.Medzhitov, R. 2001. Toll-like receptors and innate immunity. Nat. Rev. Immunol. 1:135-145.

111.Tian, J., et al. 2007. Toll-like receptor 9-dependent activation by DNA-containing immune complexes is mediated by HMGB1 and RAGE. Nat. Immunol. 8:487-496.

112.van Zoelen, M.A., et al. 2009. Role of toll-like receptors 2 and 4, and the receptor for advanced glycation end products in high-mobility group box 1induced inflammation in vivo. Shock. 31:280-284.

113.Basu, S., and Fenton, M.J. 2004. Toll-like receptors: function and roles in lung disease. Am. J. Physiol. Lung Cell. Mol. Physiol. 286:L887-L892.

114.Baumgarten, G., et al. 2001. In vivo expression of proinflammatory mediators in the adult heart after endotoxin administration: the role of toll-like receptor-4. J. Infect. Dis. 183:1617-1624.

115. Chakravarty, S., and Herkenham, M. 2005. Toll-like receptor 4 on nonhematopoietic cells sustains CNS inflammation during endotoxemia, independent of systemic cytokines. J. Neurosci. 25:1788-1796.

116.Matsumura, T., Ito, A., Takii, T., Hayashi, H., and Onozaki, K. 2000. Endotoxin and cytokine regula- tion of toll-like receptor (TLR) 2 and TLR4 gene expression in murine liver and hepatocytes. J. Interferon Cytokine Res. 20:915-921.

117. Tsuboi, N., et al. 2002. Roles of toll-like receptors in C-C chemokine production by renal tubular epithelial cells. J. Immunol. 169:2026-2033.

118.Leemans, J.C., et al. 2005. Renal-associated TLR2 mediates ischemia/reperfusion injury in the kidney. J. Clin. Invest. 115:2894-2903.

119. Wolfs, T.G., et al. 2002. In vivo expression of Tolllike receptor 2 and 4 by renal epithelial cells: IFNgamma and TNF-alpha mediated up-regulation during inflammation. J. Immunol. 168:1286-1293.

120.El-Achkar, T.M., et al. 2006. Sepsis induces changes in the expression and distribution of Toll-like receptor 4 in the rat kidney. Am. J. Physiol. Renal Physiol. 290:F1034-F1043.

121.Feterowski, C., et al. 2003. Effects of functional Toll-like receptor-4 mutations on the immune response to human and experimental sepsis. Immunology. 109:426-431.

122. Weighardt, H., et al. 2002. Cutting edge: myeloid differentiation factor 88 deficiency improves resistance against sepsis caused by polymicrobial infection. J. Immunol. 169:2823-2827.

123.McMasters, K.M., Peyton, J.C., Hadjiminas, D.J., and Cheadle, W.G. 1994. Endotoxin and tumour necrosis factor do not cause mortality from caecal ligation and puncture. Cytokine. 6:530-536.

124. Roger, T., et al. 2009. Protection from lethal gramnegative bacterial sepsis by targeting Toll-like receptor 4. Proc. Natl. Acad. Sci. U. S. A. 106:2348-2352.

125.Peck-Palmer, O.M., et al. 2008. Deletion of MyD88 markedly attenuates sepsis-induced T and B lymphocyte apoptosis but worsens survival. J. Lenkoc. Biol. 83:1009-1018. 